\title{
CLASH-VLT: spectroscopic confirmation of a $z=6.11$ quintuply lensed galaxy in the Frontier Fields cluster RXC J2248.7-4431 ${ }^{\star}$
}

\author{
I. Balestra' ${ }^{1,2}$, E. Vanzella ${ }^{3}$, P. Rosati ${ }^{4}$, A. Monna ${ }^{5}$, C. Grillo ${ }^{6}$, M. Nonino ${ }^{1}$, A. Mercurio ${ }^{2}$, A. Biviano ${ }^{1}$, L. Bradley ${ }^{7}$, \\ D. $\mathrm{Coe}^{7}$, A. Fritz ${ }^{8}$, M. Postman ${ }^{7}$, S. Seitz ${ }^{5,10}$, M. Scodeggio ${ }^{8}$, P. Tozzi ${ }^{9}$, W. Zheng ${ }^{11}$, B. Ziegler ${ }^{12}$, A. Zitrin ${ }^{13}$, \\ M. Annunziatella ${ }^{14,1}$, M. Bartelmann ${ }^{13}$, N. Benitez ${ }^{15}$, T. Broadhurst ${ }^{16}$, R. Bouwens $^{17}$, O. Czoske ${ }^{12}$, \\ M. Donahue ${ }^{18}$, H. Ford ${ }^{11}$, M. Girardi ${ }^{14,1}$, L. Infante ${ }^{24}$, S. Jouvel ${ }^{23}$, D. Kelson ${ }^{19}$, A. Koekemoer ${ }^{7}$, \\ U. Kuchner ${ }^{12}$, D. Lemze ${ }^{11}$, M. Lombardi ${ }^{21}$, C. Maier ${ }^{12}$, E. Medezinski ${ }^{22}$, P. Melchior ${ }^{25}$, M. Meneghetti ${ }^{3,20}$, \\ J. Merten ${ }^{26}$, A. Molino ${ }^{15}$, L. Moustakas ${ }^{26}$, V. Presotto ${ }^{14}$, R. Smit ${ }^{17}$, and K. Umetsu ${ }^{27}$
}

(Affiliations can be found after the references)

Received 6 September 2013 / Accepted 17 October 2013

\begin{abstract}
We present VIsible Multi-Object Spectrograph (VIMOS) observations of a $z \sim 6$ galaxy quintuply imaged by the Frontier Fields galaxy cluster RXC J2248.7-4431 $(z=0.348)$. This sub- $L^{*}$, high- $z$ galaxy has been recently discovered by Monna et al. (2013) using dropout techniques with the 16-band HST photometry acquired as part of the Cluster Lensing And Supernova survey with Hubble (CLASH). Obtained as part of the CLASH-VLT survey, the VIMOS medium-resolution spectra of this source show a very faint continuum between $\sim 8700 \AA$ and $\sim 9300 \AA$ and a prominent emission line at $8643 \AA$, which can be readily identified with Lyman- $\alpha$ at $z=6.110 \pm 0.002$. The emission line exhibits an asymmetric profile, with a more pronounced red wing. The rest-frame equivalent width of the line is $E W=79 \pm 10 \AA$, relatively well constrained thanks to the detection of the UV continuum, which is rarely achieved for a sub- $L^{*}$ galaxy at this redshift. After correcting for magnification, the star formation rate (SFR) estimated from the $\operatorname{Ly} \alpha$ line is $S F R(\operatorname{Ly} \alpha)=11 M_{\odot} \mathrm{yr}^{-1}$ and that estimated from the UV data is $S F R(\mathrm{UV})=3 M_{\odot} \mathrm{yr}^{-1}$. We estimate that the effective radius of the source is $R_{\mathrm{e}} \lesssim 0.4 \mathrm{kpc}$, which implies a star formation surface mass density $\Sigma_{\mathrm{SFR}}>6 M_{\odot} \mathrm{yr}^{-1} \mathrm{kpc}^{-2}$ and, using the Kennicutt-Schmidt relation, a gas surface mass density $\Sigma_{\text {gas }}>10^{3} M_{\odot} \mathrm{pc}^{-2}$. Our results support the idea that this magnified, distant galaxy is a young and compact object with luminosity $0.4 L^{*}$ at $z=6$, when the Universe was just 1 Gyr old, with a similar amount of mass in gas and stars. In the spirit of the Frontier Fields initiative, we also publish the redshifts of several multiply imaged sources and other background objects, which will help improving the strong-lensing model of this galaxy cluster.
\end{abstract}

Key words. gravitational lensing: strong - galaxies: high-redshift

\section{Introduction}

Understanding the process of reionization of the intergalactic medium in the early Universe and the nature of the first galaxies responsible for that process are among the most important goals of modern cosmology (e.g. Robertson et al. 2010, and references therein). In recent years, great progress has been made in our ability to detect galaxies at $z \gtrsim 6$. To the greatest extent, this has been possible thanks to very deep observations with the Hubble Space Telescope (HST). The selection of optical dropouts in deep HST fields has led to the identification of samples that now reach hundreds of candidate Lyman-break galaxies (LBG) at $z \sim 6-8$ (e.g. Bouwens et al. 2006, 2011; Koekemoer et al. 2011; Trenti et al. 2012; Oesch et al. 2012; Yan et al. 2012; Bradley et al. 2012, 2013; Ellis et al. 2013).

Acting as cosmic telescopes, massive lensing galaxy clusters allow the identification of faint, otherwise undetected, high- $z$ galaxies. Despite the smaller volumes probed with gravitational lensing compared to those probed in deep fields, lensing has been successful in pushing the detection of distant galaxies one step farther, as testified by the recent discoveries of

* This work is based on data collected at ESO VLT (prog.ID 186.A0798) and at NASA HST. several $z \sim 9-11$ candidates behind massive clusters (Bouwens et al. 2012; Zheng et al. 2012; Coe et al. 2013). All of these high- $z$ candidates have been identified by galaxy clusters observed as part of the Cluster Lensing And Supernova survey with Hubble (CLASH; Postman et al. 2012) Multi-Cycle Treasury program.

Ground-based spectroscopy of high-redshift sources in "blank" fields can be very challenging given that they are often 27 th magnitude or fainter. However, the flux magnifications due to gravitational lensing helps to bring these distant sources within reach of ground-based spectroscopy. Lensed observations reach significantly lower luminosities (e.g. Schenker et al. 2012) than spectroscopic observations in deep fields (e.g. Vanzella et al. 2011).

The most prominent spectral feature in the UV rest-frame wavelengths probed by optical/near-infrared spectroscopy at $z \gtrsim$ 6 is the $\operatorname{Lyman}-\alpha(\operatorname{Ly} \alpha)$ emission line. The $\operatorname{Ly} \alpha$ emission line is a resonant transition and suffers from radiative transfer effects, a property that further complicates the modeling of the escape fraction of Ly $\alpha$ photons from their host galaxies (Verhamme et al. 2006; Dijkstra \& Jeeson-Daniel 2013). The line itself is also an important diagnostic of the physical processes at work (Giavalisco et al. 1996; Vanzella et al. 2009), since its strength and velocity profile depend on the instantaneous star formation 


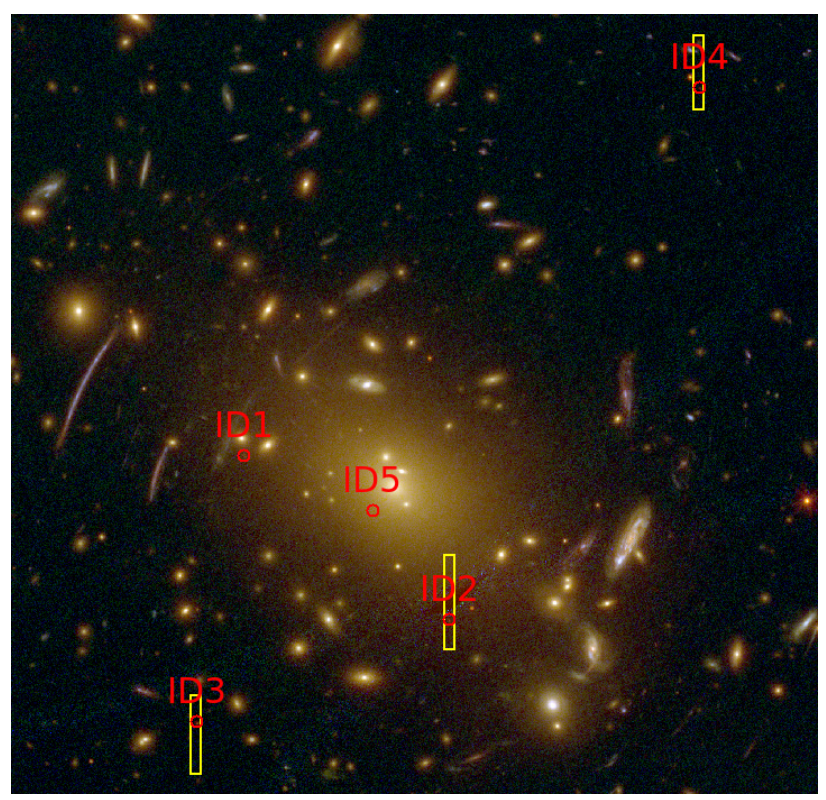

Fig. 1. HST color-composite image. The red circles (ID1-5) show the positions of the quintuply lensed dropout candidate at $z=6$, labeled as in M13. The three VIMOS slits (yellow boxes) were placed on ID2, ID3, and ID4 in four repeated 1h-pointings. ID1 was not targeted because it is too close to a bright cluster-member galaxy.

rate, gas and dust content, metallicity, kinematics, and geometry of the interstellar medium (e.g., clumpy, anisotropic).

The cluster RXC J2248.7-4431 ( $z=0.348)$ was observed as part of the CLASH-VLT large spectroscopic program, which targets 14 CLASH clusters in the southern sky. Five multiple images of a young galaxy, identified as $i$-dropouts, have been recently discovered from the CLASH HST imaging of this cluster (see Monna et al. 2013, hereafter M13). Two of the brightest images (ID2 and ID3) also have Spitzer detections, which additionally support the high- $z$ nature of the source (see M13). The delensed UV luminosity of the magnified source, inferred from the strong-lensing analysis is $L_{1600} \sim 0.4 L_{1600}^{*}$ at $z=6$. The UV slope is steep $(\beta=-2.89 \pm 0.25)$ and the inferred age $(<300 \mathrm{Myr})$, mass $\left(\sim 10^{8} M_{\odot}\right)$, and metallicity $\left(Z<0.2 Z_{\odot}\right)$ are all consistent with that of a young galaxy (M13).

In this Letter, we report on our VIMOS/VLT observations, which provide spectroscopic confirmation at $z=6.110$ for three of the high-redshift, quintuply imaged $i$-dropouts in RXC J2248.7-4431, and we infer some of the physical properties of this young galaxy. In addition, we provide a list of redshift measurements for several other strong-lensing features and magnified background sources targeted in this cluster by our CLASH-VLT survey so far. Errors are quoted to the $1 \sigma$ confidence level, unless otherwise stated. We assume a cosmology with $\Omega_{\mathrm{tot}}, \Omega_{\mathrm{M}}, \Omega_{\Lambda}=1.0,0.3,0.7$ and $H_{0}=70 \mathrm{~km} \mathrm{~s}^{-1} \mathrm{Mpc}^{-1}$.

\section{VIMOS observations and data reduction}

The cluster RXC J2248.7-4431 was observed in June-July 2013. The VIMOS data were acquired using four separate pointings with one quadrant centered on the cluster core. Three of the five multiple images of the $i$-dropout candidate were targeted in four medium-resolution (MR) 1h-pointings, providing us a total of $4 \mathrm{~h}$ of integration time on each of the three images. The three multiple images targeted are displayed in Fig. 1. The identification numbers of the three targets are those used in M13.

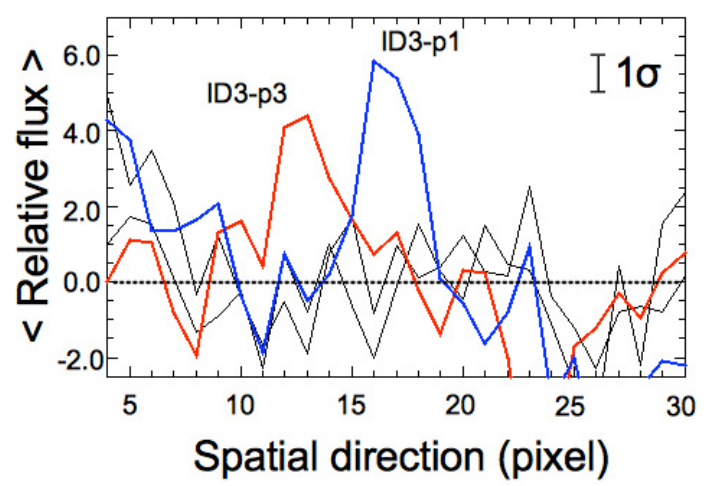

Fig. 2. Spatial profiles along the direction of the slit for the spectra of ID3, derived by collapsing counts in the dispersion direction between $9050 \AA$ and $9280 \AA$ for each pointing separately. P1 is plotted in blue, $\mathrm{P} 3$ in red, and $\mathrm{P} 2$ and $\mathrm{P} 4$ in black. The continuum is clearly detected in $\mathrm{P} 1(\sim 5 \sigma)$ and $\mathrm{P} 3(\sim 3 \sigma)$. Spectra are generally at slightly different pixel positions in different pointings.

The masks were designed with $1^{\prime \prime}$-slits and the VIMOS spatial resolution is $0.205^{\prime \prime} /$ pixel. The MR grism and the GG475 filter were used. In this configuration the useable wavelength range is 4800-10000 $\AA$, the resolution is $R=580$ (or $\sim 13 \AA$ ), and the dispersion is $2.55 \AA$ /pixel.

Data reduction was performed using the Vimos Interactive Pipeline Graphical Interface (VIPGI; Scodeggio et al. 2005), which uses standard automated procedures to compute bias subtraction, flat-fielding, sky subtraction, and wavelength calibration. The standard star EG-274 was used for flux calibration.

The seeing varied from pointing to pointing. The best seeing conditions $\left(\sim 0.6-0.7^{\prime \prime}\right)$ were reached during pointing P1 and P3 (July 11 and 10), while they were poorer $\left(\sim 1.0-1.1^{\prime \prime}\right)$ during pointing P2 and P4 (July 6 and 9). As a consequence of the varying seeing conditions the signal-to-noise ratio $(\mathrm{S} / \mathrm{N})$ of the spectra varied significantly in the different pointings.

\section{Results}

A strong emission line is detected at $8643 \AA$ in each of the single 1h-spectra (P1-P4) for all of the three multiple images targeted (ID2-ID4). If the emission line is identified to be Ly $\alpha$, the redshift of the source is $z=6.110$, which is consistent both with the photo-z $\left(z_{\text {phot }} \simeq 5.9\right.$, see M13) and with the strong-lensing model predictions ( $z_{\text {lensing }} \simeq 6.0$, see M13). For the brightest image (ID3), in the two pointings with better seeing (P1 and P3) a faint continuum is also detected (at about $5 \sigma$ ) between $\sim 8700 \AA$ and $\sim 9300 \AA$ (see Fig. 2). To increase the $\mathrm{S} / \mathrm{N}$, we tried to stack the four 1h-spectra for each multiple image. However, the $\mathrm{S} / \mathrm{N}$ is maximized when only the two spectra with the highest $\mathrm{S} / \mathrm{N}$ ( $\mathrm{P} 1$ and $\mathrm{P} 3$ ) are combined. The higher $\mathrm{S} / \mathrm{N}$ in these two pointings is due to a combination of better seeing and better transparency at the time of the observations. Figure 3 shows the sum of the 2D spectra for each of the three multiple images (ID2, ID3, and ID4) and the highest S/N combined spectrum (2h-stacked spectrum of ID3). Unfortunately, even in the stacked spectrum the $\mathrm{S} / \mathrm{N}$ of the continuum is not sufficiently high to reliably identify any other UV absorption feature. It is worth to recall that star-forming galaxies (and their stacks) with a large Ly $\alpha$ equivalent width $(E W>50 \AA)$ show very weak or even lack UV stellar and interstellar absoption lines (e.g., Vanzella et al. 2009; Balestra et al. 2010), therefore it could be intrinsically 


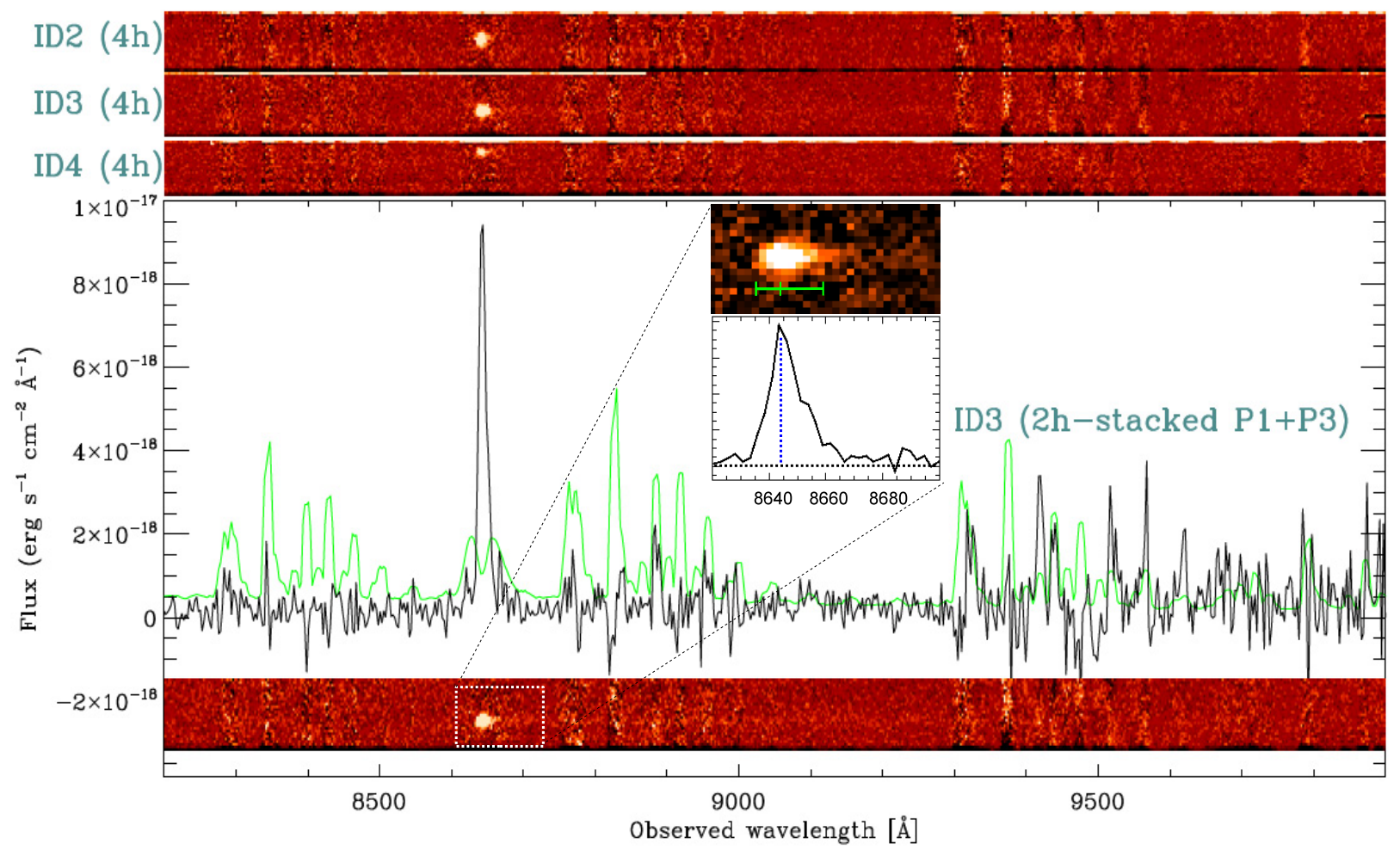

Fig. 3. VIMOS 1D spectrum of ID3 (2h-exposure, pointing P1+P3; black line) and of the sky rescaled to arbitrary units (green line). The lower inset shows the sum of the 2D spectra $(2 \times 1 \mathrm{~h}$ exposure, $\mathrm{P} 1+\mathrm{P} 3)$. The $4 \mathrm{~h}$-stacked $2 \mathrm{D}$ spectra of each multiple image (ID2, ID3, and ID4) are shown in the insets on the top. A zoomed version of the 1D and 2D spectra around the Ly $\alpha$ is shown in the central inset. The vertical blue dotted line marks the position of the peak of the line, which shows a clearly asymmetric profile. In the 2D spectrum, the green segments mark the position of the peak of the flux and that of the $20 \%$ flux level.

difficult to detect them even in higher $\mathrm{S} / \mathrm{N}$ spectra. The lack of the $\mathrm{N}$ V emission line suggests the absence of strong nuclear activity (AGN), corroborated by the lack of X-ray detection. However, since the $2 \sigma$ upper limit on the $0.5-10 \mathrm{keV}$ rest-frame luminosity is only $5 \times 10^{44} \mathrm{erg} \mathrm{s}^{-1}$ (obtained by stacking Chandra counts at the position of the three brightest magnified images), a low-luminosity AGN cannot be excluded with the current shallow X-ray.

To obtain a more precise measurement of the continuum flux and, hence, a more reliable estimate of the EW of the Ly $\alpha$, we used only the highest S/N spectrum (ID3 2h-stacked spectrum). The integrated flux of the line is $1.63 \times 10^{-16} \mathrm{erg} \mathrm{s}^{-1} \mathrm{~cm}^{-2}$ and the continuum has a flux of $2.9 \times 10^{-19} \mathrm{erg} \mathrm{s}^{-1} \mathrm{~cm}^{-2} \AA^{-1}$ in the wavelength range $\sim 9000-9300 \AA$. This yields an equivalent width of $E W=79 \pm 10 \AA$ for the Ly $\alpha$, where the error is estimated from the statistical error on the flux of the continuum.

The line profile is asymmetric with a more pronounced red wing (see inset of Fig. 3). The asymmetry of the line is typically observed at these redshifts and in this case is mainly due to the intergalactic medium, which at $z \sim 6$ can completely absorb half of the line (e.g. Laursen et al. 2009). Other processes can shape the line profile, but the spectral resolution of our observational setup prevents us from separating possible secondary features related to internal kinematics (e.g., outflows).

We derived the star formation rate (SFR), following Kennicutt (1998), by converting the observed nebular (Ly $\alpha$ ) and

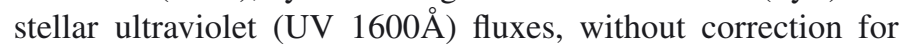
dust attenuation. After correcting for the magnification factor $(\mu=6)$, the SFR is $\operatorname{SFR}(\operatorname{Ly} \alpha)=11 M_{\odot} \mathrm{yr}^{-1}$ and $\operatorname{SFR}(\mathrm{UV})=$ $3 M_{\odot} \mathrm{yr}^{-1}$, respectively. The intrinsic $\operatorname{SFR}(\operatorname{Ly} \alpha)$ can be even higher (possibly double) since the IGM most probably attenuates $\sim 50 \%$ of the line flux. Interestingly, the $\operatorname{SFR}(\operatorname{Ly} \alpha)$ is higher, or at the most similar to that derived from the UV continuum. As discussed in Verhamme et al. (2008), this is indicative of very low or negligible dust attenuation coupled with very young stellar populations and recent onset of star formation activity (consistent with recent results by M13). Given the stellar mass derived from SED fitting $\left(\sim 2 \times 10^{8} M_{\odot}\right.$, see M13), the specific star formation rate is $\operatorname{sSFR}(\operatorname{Ly} \alpha)=55 \mathrm{Gyr}^{-1}$ (or $\mathrm{s} S F R(\mathrm{UV})=$ $15 \mathrm{Gyr}^{-1}$ ).

To estimate the size of the source we measured the effective radii of ID2, ID3, and ID4 from the HST images and the magnification factors obtained from the lensing model by M13. We estimate that the effective radius of the source is $R_{\mathrm{e}} \lesssim 0.4 \mathrm{kpc}$. Given our estimate of the size and the value of SFR(UV), we can compute the SFR surface mass density $\Sigma_{\mathrm{SFR}}>6 M_{\odot} \mathrm{yr}^{-1} \mathrm{kpc}^{-2}$ and we can use the Kennicutt-Schmidt relation to derive the gas surface mass density $\Sigma_{\text {gas }}>10^{3} \mathrm{M}_{\odot} \mathrm{pc}^{-2}$. This indicates that the stellar-over-gas mass ratio is close to unity, in line with theoretical expectations for small mass $\left(\lesssim 10^{9} M_{\odot}\right)$ and low SFR objects at these redshifts (see Calura et al. 2008).

\subsection{Redshifts of arcs and other background objects}

As part of our CLASH-VLT survey, we targeted several multiply imaged sources and candidate high- $z$ objects. In Table 1, we report the spectroscopic redshifts obtained so far for this cluster also with the low-resolution blue grism. We confirm 10 of the 14 multiple image systems used by M13. These measurements confirm the high accuracy of the photometric redshifts and additionally support the strong-lensing $\operatorname{model}\left(z_{\text {lens }} \simeq z_{\text {spec }}\right)$. 
Table 1. Redshifts of 10 multiple image systems and other background objects measured in the core of RXC J2248.7-4431 to date.

\begin{tabular}{lcccc}
\hline \hline $\begin{array}{l}\text { RA } \\
(1)\end{array}$ & $\begin{array}{c}\text { Dec } \\
(2)\end{array}$ & $\begin{array}{c}z_{\text {spec }} \\
(3)\end{array}$ & $\begin{array}{c}z_{\text {phot }} \\
(4)\end{array}$ & $\begin{array}{c}z_{\text {lens }} \\
(5)\end{array}$ \\
\hline $22: 48: 43.45$ & $-44: 32: 04.6$ & 6.110 & $5.87_{-0.02}^{+0.03}$ & $6.0($ ID2) \\
$22: 48: 45.81$ & $-44: 32: 14.8$ & 6.110 & $6.01_{-0.06}^{+0.03}$ & 6.0 (ID3) \\
$22: 48: 41.11$ & $-44: 31: 11.4$ & 6.110 & $5.95_{-0.08}^{+0.06}$ & $6.0($ ID4) \\
$22: 48: 47.00$ & $-44: 31: 44.0$ & 1.229 & $1.26_{-0.03}^{+0.03}$ & $1.19(1.1 \mathrm{a})$ \\
$22: 48: 44.75$ & $-44: 31: 16.3$ & 1.229 & $1.22_{-0.02}^{+0.05}$ & $1.19(1.1 \mathrm{c})$ \\
$22: 48: 46.22$ & $-44: 31: 50.6$ & 1.260 & $1.23_{-0.06}^{+0.06}$ & $1.26(3 \mathrm{a})$ \\
$22: 48: 45.08$ & $-44: 31: 38.4$ & 1.398 & $1.53_{-0.02}^{+0.02}$ & $1.40(4 \mathrm{~b})$ \\
$22: 48: 43.01$ & $-44: 31: 24.9$ & 1.398 & $1.11_{-0.02}^{+0.02}$ & $1.40(4 \mathrm{c})$ \\
$22: 48: 45.22$ & $-44: 32: 24.0$ & 1.429 & $1.17_{-0.09}^{+0.10}$ & $1.46(6 \mathrm{a})$ \\
$22: 48: 41.56$ & $-44: 32: 23.9$ & 3.110 & $3.03_{-0.05}^{+0.05}$ & $2.97(11 \mathrm{~b})$ \\
$22: 48: 41.38$ & $-44: 32: 28.4$ & 1.270 & $1.18_{-0.07}^{+0.04}$ & - \\
$22: 48: 46.92$ & $-44: 32: 49.4$ & 1.353 & $1.18_{-0.03}^{+0.02}$ & - \\
$22: 48: 39.02$ & $-44: 32: 34.6$ & 3.242 & $3.44_{-0.07}^{+0.07}$ & - \\
$22: 48: 49.28$ & $-44: 30: 55.8$ & 3.542 & $3.55_{-0.07}^{+0.19}$ & - \\
$22: 48: 44.23$ & $-44: 31: 31.0$ & 0.730 & $0.70_{-0.03}^{+0.03}$ & - \\
\hline
\end{tabular}

Notes. (1, 2) J2000 coordinates; (3) spectroscopic redshift; (4, 5) photometric and lensing-model predicted redshift (and IDs) from M13.

\section{Discussion}

We presented VIMOS/VLT observations providing a spectroscopic confirmation at $z=6.110$ for a galaxy quintuply imaged by the Frontier Fields cluster RXC J2248.7-4431.

The VIMOS spectra clearly show a strong Ly $\alpha$ and a relatively faint, but significantly detected $(5 \sigma)$, continuum. This is a rare case where the detection of the continuum can be achieved in galaxies with delensed magnitudes of $\sim 27$. Remarkably, this was achieved in only $1 \mathrm{~h}$ of exposure. Our results, together with those recently presented by M13, suggest that this magnified, distant galaxy is a young $\left(<300 \mathrm{Myr}, \sim 10^{8} M_{\odot}\right.$, and $\left.Z<0.2 Z_{\odot}\right)$ and compact $(\lesssim 0.4 \mathrm{kpc})$ object fainter than $L^{*}$ at $z=6$, with a similar amount of mass in gas and stars. We can rule out the presence of a strong AGN because of the absence of $\mathrm{N} V$ emission line in the VIMOS spectra and because of the Chandra upper limit on the X-ray luminosity $\left(<5 \times 10^{44} \mathrm{erg} \mathrm{s}^{-1}\right)$.

While this paper was finalized, Boone et al. (2013) very recently reported the detection of a submillimeter source with APEX/LABOCA at $870 \mu \mathrm{m}$, which they tentatively associated with the $z \sim 6$ multiple system, or alternatively with a second interacting galaxy, or even with the SZ signal from the ICM. The association of the submm source with the $z \sim 6$ multiply imaged galaxy remains extremely uncertain due to the poor resolution of submm observations and to foreground contamination. The SZ explanation or the two-source hypothesis seem more plausible, because in the single-source scenario it would be very difficult to explain how the dust emission in the submillimeter $\left(A_{v} \sim 1.5\right)$ can be reconciled with the extremely steep UV spectral slope derived from CLASH photometry $(\beta \sim-2.9$, see M13) and the prominent Ly $\alpha$ emission, $E W \simeq 80 \AA$. Both features typically indicate very low dust attenuation (Hayes et al. 2011; de Barros et al. 2012; Atek et al. 2013; Laursen et al. 2013). Therefore, we interpret the HST $z=6.110$ source as a young and compact object with low dust content in an early phase of evolution, when the Universe was just 1 Gyr old. High spatial-resolution submillimiter observations with ALMA will be extremely useful to reliably constrain the SFR and the molecular gas content in this magnified high- $z$ system.

Acknowledgements. We thank the anonymous referee for the valuable comments and suggestions. We acknowledge partial support by the DFG Cluster of Excellence Origin Structure of the Universe. A.Z. is supported by contract research "Internationale Spitzenforschung II/2-6" of the Baden-Württemberg Stiftung.

\section{References}

Atek, H., Kunth, D., Schaerer, D., et al. 2013, A\&A, in press, DOI: $10.1051 / 0004-6361 / 201321519$

Balestra, I., Mainieri, V., Popesso, P., et al. 2010, A\&A, 512, A12

Boone, F., Clément, B., Richard, J., et al. 2013, A\&A, 559, L1

Bouwens, R., Bradley, L., Zitrin, A., et al. 2012, ApJ, submitted [arXiv: 1211.2230]

Bouwens, R. J., Illingworth, G. D., Blakeslee, J. P., \& Franx, M. 2006, ApJ, 653, 53

Bouwens, R. J., Illingworth, G. D., Oesch, P. A., et al. 2011, ApJ, 737, 90

Bradley, L. D., Trenti, M., Oesch, P. A., et al. 2012, ApJ, 760, 108

Bradley, L. D., Zitrin, A., Coe, D., et al. 2013, ApJ, submitted [arXiv: 1308.1692]

Calura, F., Jimenez, R., Panter, B., Matteucci, F., \& Heavens, A. F. 2008, ApJ, 682,252

Coe, D., Zitrin, A., Carrasco, M., et al. 2013, ApJ, 762, 32

de Barros, S., Schaerer, D., \& Stark, D. P. 2012, A\&A, submitted [arXiv: 1207.3663]

Dijkstra, M., \& Jeeson-Daniel, A. 2013, MNRAS, 435, 3333

Ellis, R. S., McLure, R. J., Dunlop, J. S., et al. 2013, ApJ, 763, L7

Giavalisco, M., Koratkar, A., \& Calzetti, D. 1996, ApJ, 466, 831

Hayes, M., Schaerer, D., Östlin, G., et al. 2011, ApJ, 730, 8

Koekemoer, A. M., Faber, S. M., Ferguson, H. C., et al. 2011, ApJS, 197, 36

Laursen, P., Sommer-Larsen, J., \& Andersen, A. C. 2009, ApJ, 704, 1640

Laursen, P., Duval, F., \& Östlin, G. 2013, ApJ, 766, 124

Monna, A., Seitz, S., Greisel, N., et al. 2013, MNRAS, submitted [M13] [arXiv: 1308.6280$]$

Oesch, P. A., Bouwens, R. J., Illingworth, G. D., et al. 2012, ApJ, 759, 135

Postman, M., Coe, D., Benítez, N., et al. 2012, ApJS, 199, 25

Robertson, B. E., Ellis, R. S., Dunlop, J. S., McLure, R. J., \& Stark, D. P. 2010, Nature, 468, 49

Schenker, M. A., Stark, D. P., Ellis, R. S., et al. 2012, ApJ, 744, 179

Scodeggio, M., Franzetti, P., Garilli, B., et al. 2005, PASP, 117, 1284

Trenti, M., Bradley, L. D., Stiavelli, M., et al. 2012, ApJ, 746, 55

Vanzella, E., Giavalisco, M., Dickinson, M., et al. 2009, ApJ, 695, 1163

Vanzella, E., Pentericci, L., Fontana, A., et al. 2011, ApJ, 730, L35

Verhamme, A., Schaerer, D., \& Maselli, A. 2006, A\&A, 460, 397

Verhamme, A., Schaerer, D., Atek, H., \& Tapken, C. 2008, A\&A, 491, 89

Yan, H., Finkelstein, S. L., Huang, K.-H., et al. 2012, ApJ, 761, 177

Zheng, W., Postman, M., Zitrin, A., et al. 2012, Nature, 489, 406

1 INAF - Osservatorio Astronomico di Trieste, via G. B. Tiepolo 11, 34131 Trieste, Italy

e-mail: italobale@gmail.com

2 INAF - Osservatorio Astronomico di Capodimonte, via Moiariello 16, 80131 Napoli, Italy

3 INAF - Osservatorio Astronomico di Bologna, via Ranzani 1, 40127 Bologna, Italy

4 Dipartimento di Fisica e Scienze della Terra, Università di Ferrara, via Saragat 1, 44122 Ferrara, Italy

5 University Observatory Munich, Scheinerstrasse 1, 81679 München, Germany

6 Dark Cosmology Centre, Niels Bohr Institute, University of Copenhagen, Juliane Maries Vej 30, 2100 Copenhagen, Denmark

7 Space Telescope Science Institute, 3700 San Martin Drive, Baltimore, MD 21218, USA

8 INAF/IASF-Milano, via Bassini 15, 20133 Milano, Italy

9 INAF - Osservatorio Astrofisico di Arcetri, Largo E. Fermi 5, 50125 Firenze, Italy

10 Max-Planck-Institut für extraterrestrische Physik, Postfach 1312, Giessenbachstr., 85741 Garching, Germany

11 Department of Physics and Astronomy, The Johns Hopkins University, 3400 North Charles Street, Baltimore, MD 21218, USA 
I. Balestra et al.: CLASH-VLT: spectroscopic confirmation of $z=6.11$ lensed galaxy

12 University of Vienna, Department of Astrophysics, Türkenschanzstr. 17, 1180 Wien, Austria

13 Universität Heidelberg, Philosophenweg 12, 69120 Heidelberg, Germany

14 Dipartimento di Fisica, Università degli Studi di Trieste, via Tiepolo 11, 34143 Trieste, Italy

15 Instituto de Astrofísica de Andalucía (CSIC), C/Camino Bajo de Huétor 24, 18008 Granada, Spain

16 Department of Theoretical Physics, University of the Basque Country, PO Box 644, 48080 Bilbao, Spain

17 Leiden Observatory, Leiden University, PO Box 9513, 2300 RA Leiden, The Netherlands

18 Department of Physics and Astronomy, Michigan State University, East Lansing, MI 48824, USA

19 Observatories of the Carnegie Institution of Washington, Pasadena, CA 91 101, USA
${ }^{20}$ INFN - Bologna, via Ranzani 1, 40127 Bologna, Italy

21 Dipartimento di Fisica, Università degli Studi di Milano, via Celoria 16, 20133 Milan, Italy

22 Department of Physics and Astronomy, The Johns Hopkins University, 3400 North Charles Street, Baltimore, MD 21218, USA

23 Institut de Ciències de l'Espai (IEEC-CSIC), 08193 Bellaterra (Barcelona), Spain

24 Departamento de Astronomia y Astrofisica, Pontificia Universidad Catolica de Chile, V. Mackenna 4860, 22 Santiago, Chile

25 Department of Physics, The Ohio State University, Columbus, $\mathrm{OH}$, USA

26 Jet Propulsion Laboratory, California Institute of Technology, 4800 Oak Grove Dr, Pasadena, CA 91109, USA

27 Institute of Astronomy and Astrophysics, Academia Sinica, PO Box 23-141, 10617 Taipei, Taiwan 